\title{
Optical full Hadamard matrix multiplexing and noise effects
}

\author{
L. Streeter,${ }^{1,2, \star}$ G. R. Burling-Claridge,${ }^{2}$ M. J. Cree, ${ }^{1}$ and R. Künnemeyer ${ }^{1}$ \\ 'Department of Engineering, University of Waikato, Gate 1, Knighton Road, \\ Private Bag 3105, Hamilton 3240, New Zealand \\ ${ }^{2}$ AgResearch, Ruakura Research Centre, East Street, Private Bag 3123, Hamilton 3240, New Zealand \\ ${ }^{*}$ Corresponding author: lee.streeter@agresearch.co.nz
}

Received 24 November 2008; revised 2 March 2009; accepted 3 March 2009; posted 4 March 2009 (Doc. ID 104469); published 2 April 2009

\begin{abstract}
Hadamard multiplexing provides a considerable SNR boost over additive random noise but Poisson noise such as photon noise reduces the boost. We develop the theory for full H-matrix Hadamard transform imaging under additive and Poisson noise effects. We show that $\mathrm{H}$-matrix encoding results in no effect on average on the noise level due to Poisson noise sources while preferentially reducing additive noise. We use this result to explain the wavelength-dependent varying SNR boost in a Hadamard hyperspectral imager and argue that such a preferential boost is useful when the main noise source is indeterminant or varying. (C) 2009 Optical Society of America
\end{abstract}

OCIS codes: $\quad 110.4234,110.1758,030.4280,350.6980$.

\section{Introduction}

Hadamard multiplexing is the technique of measuring groups of samples according to specific patterns to boost the signal-to-noise ratio (SNR). There are two types of Hadamard matrices commonly employed in multiplexing, namely the H-matrix and the S-matrix [1]. The H-matrix is the full matrix of positive and negative ones and size $N \times N$ that is optimal in terms of SNR boost over random noise. The S-matrix consists of ones and zeros, is of size $(N-1) \times(N-1)$ and can be derived from the $\mathrm{H}$ matrix. The S-matrix is more commonly used in multiplexing for the practical reason that the ones and zeros are optically implemented as open or closed apertures or mirror deflections towards or away from a sensor. The negative ones in the $\mathrm{H}$-matrix require special consideration to implement. The S-matrix, while not as optimal as the H-matrix (less SNR boost), is conjectured to be the optimal one-zero matrix for SNR boost over additive noise.

0003-6935/09/112078-08\$15.00/0

(C) 2009 Optical Society of America
In light multiplexing systems the Hadamard SNR boost is reduced by Poisson noise [2,3]. Indeed, if Poisson noise, such as photon noise, is the only significant noise contribution then S-matrix multiplexing actually reduces the SNR. If Poisson and additive Gaussian noise are both present then the SNR boost achieves some intermediate value less than the theoretical best. Schemes have been proposed to boost the SNR under Poisson noise [4] and to optimally boost with a trade-off between Poisson and Gaussian noise [5].

Hadāmard transform imaging was traditionally implemented using the S-matrix via mechanical gratings with holes and occlusions [1]. Examples include rectangular gratings that moved laterally, rotating disks, and combinations thereof. Reflections and occlusions have been used to implement the H-matrix [6]. More recently imagers and imaging spectrometers based on the Texas Instruments digital micromirror device (DMD) have been described. A variety of modalities have been investigated using DMDs and single diode sensors [7], linear sensor array dispersive spectrometers [ $[\underline{8}, \overline{9}]$, and two-dimensional sensor arrays such as charge-coupled devices and diode arrays [10-12]. 
The Hadamard transform has been applied to the spatial and/or spectral dimension(s). Modulation of the light has been implemented before [7-9] and after the sample [10-12]. The compressed sensing single pixel camera architecture $[13,14]$ is a related technique. A DMD-based optical arrangement multiplexes image pixels according to a random pattern after the sample. The multiplexed signal is measured by a single light sensor. The coefficients of a linear transformation of the image are then computed from which the image is reconstructed.

We present here the theoretical development of H-matrix encoding for multiplexed imaging. The treatment is sufficiently general that it can be applied to nonimaging multiplexing systems. We include random noise sources, including additive random Gaussian instrument noise and multiplicative Poisson photon noise. We also consider the correction of systematic additive and multiplicative error. Of particular interest here is the effect of photon noise under H-matrix multiplexing with illumination drift. We present experimentation to determine the SNR boost for our optical spatially multiplexed hyperspectral imager. The SNR boost is evaluated, and the developed theory is used to explain the observed behavior of our system. The usability of the acquired spectra for practical applications is demonstrated.

\section{Full Hadamard Multiplexing under Photon and Instrument Noise}

In this section we present the theoretical development of Hadamard encoding in the presence of noise. First we review the derivation of the well-known result for the Hadamard advantage in the presence of random additive noise only. Then we extend the analysis to include a complement encoding implementation of full $\mathrm{H}$-matrix Hadamard encoding and include Poisson noise effects. Finally we include the effect of reference beam correction in the analysis.

\section{A. Background to Multiplexing}

Consider Hadamard encoding with random additive noise only. Hadamard multiplex imaging proceeds by measuring groups of pixels according to specific patterns. The patterns are the Hadamard basis functions found in the rows of a Hadamard matrix [1]. A Hadamard matrix $H$ is a self-transpose $N \times \bar{N}$ square matrix of positive and negative ones that satisfies

$$
H^{T} H=H^{2}=N I,
$$

where $I$ is the identity matrix. The Hadamard matrix forms an orthogonal basis set, thus it follows that the inverse of a Hadamard matrix is

$$
H^{-1}=\frac{1}{N} H .
$$

The data to be estimated are image pixels represented by the column vector $\mathbf{p}$ containing the trans- pose of the concatenated rows. Data acquired via Hadamard multiplexing with random additive noise e can be modeled as

$$
\mathbf{a}=H \mathbf{p}+\mathbf{e} .
$$

Inversion gives the pixel estimates $\hat{\mathbf{p}}$ as

$$
\hat{\mathbf{p}}=\mathbf{p}+\frac{1}{N} H \mathbf{e}
$$

If the image is acquired one pixel at a time then the mean squared error (MSE) is $\sigma_{0}^{2}$. In contrast, the MSE of the estimates via Hadamard multiplexing $\sigma_{H}^{2}$ is given in terms of $\sigma_{0}^{2}$ as [1]

$$
\sigma_{H}^{2}=\frac{1}{N} \sigma_{0}^{2}
$$

The reduction of additive noise variance by the factor of $N$ is known as the Hadamard advantage [1].

\section{B. Encoding Procedure}

The data acquisition proceeds by sequential illumination of the sample according to the rows of the Hadamard matrix. Spatial multiplexed Hadamard hyperspectral imaging involves three dimensions of operation: data acquisition $j$, pixel index $i$, and wavelength $\lambda$. The encoding procedure is applied identically to each wavelength in parallel so the dependence on wavelength is implicitly assumed. We split the Hadamard encoding matrix $H$ into two parts: a positive part $H^{+}$and a negative part $H^{-}$such that $H=H^{+}-H^{-}$, where

$$
H^{+}=\frac{1+H}{2}, \quad H^{-}=\frac{1-H}{2} .
$$

The $H^{+}$and $H^{-}$encode complementary subsets of the pixels. Figure 1 illustrates the splitting process. Davis [6] used occlusion and reflection to split the Hadamard matrix to perform hyperspectral imaging. The optics of our system in conjunction with the splitting of the Hadamard matrix facilitates background illumination removal [8], which was not considered

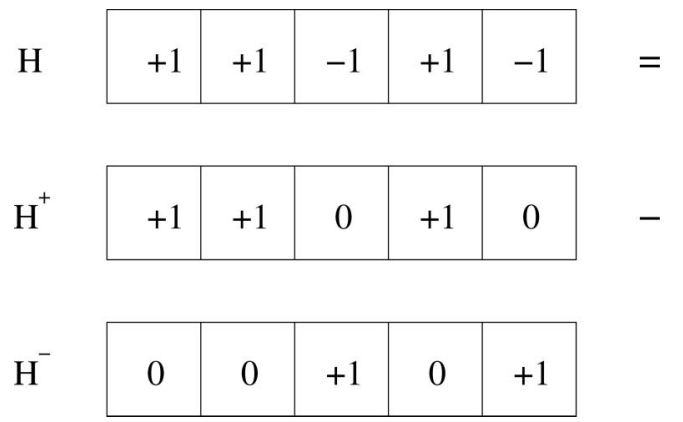

Fig. 1. Illustration of splitting the Hadamard matrix into $H^{+}$and $H^{-}$components. In $H^{+}$the -1 's of the original H-matrix are converted to 0 's. In $H^{-}$the +1 's are converted to 0 's and the -1 's are converted to +1 's. 
by Davis. The splitting process was also proposed in optical communications for code division multiple access systems [15] to boost the SNR of identifying a single user from multiple signals.

To acquire the spectra each row of $\mathrm{H}^{+}$is wrapped into a two-dimensional pattern and projected onto the sample, and the corresponding entry of $\mathbf{a}^{+}$is acquired. Immediately after each row of $\mathrm{H}^{+}$the corresponding row of $\mathrm{H}^{-}$is similarly wrapped and projected onto the sample and the corresponding entry of $\mathbf{a}^{-}$acquired. The spectra are contaminated by random additive instrument noise $\mathbf{e}$ and the additive combination of dark current, background, and stray light, all represented by $T$. The light source in our experimental setup has been shown to be stable during the data acquisition period [16]. Regardless we model the effect of light source drift during the data acquisition as multiplication by the diagonal matrix $R$. The $j$ th diagonal element in $R$ is $\langle r\rangle_{j}$ and represents the mean illumination at the time of the $j$ th acquisition. Let $\langle r\rangle$ be mean illumination over the data acquisition period, i.e., the mean of the $\langle r\rangle_{j}$. If the light source does not drift then $\langle r\rangle_{j}=\langle r\rangle$ for all $j$ and so $R=\langle r\rangle I$. The spectral parameter, $\alpha_{s}$, is introduced to represent the attenuation of the light intensity through the sample illumination optics. Likewise the parameter $\alpha_{r}$ represents the illumination attenuation through the reference beam optics. The illumination has random fluctuation following Poisson statistics represented by the diagonal matrix of random errors $E_{P}$. For pixel responses $0 \leq\{\mathbf{p}\}_{i} \leq 1$ at pixel $i$ on the sample, the acquired spectra are then

$$
\begin{aligned}
& \mathbf{a}^{+}=\alpha_{s}\left(R^{+}+E_{P}^{+}\right) H^{+} \mathbf{p}+T+\mathbf{e}^{+}, \\
& \mathbf{a}^{-}=\alpha_{s}\left(R^{-}+E_{P}^{-}\right) H^{-} \mathbf{p}+T+\mathbf{e}^{-} .
\end{aligned}
$$

The variance of the diagonal entries in $E_{p}$ is

$$
\operatorname{var}\left(\left\{E_{P}\right\}_{j, j}\right)=\{R\}_{j, j}=\langle r\rangle_{j}
$$

where $\operatorname{var}(\cdot)$ denotes variance. Equation (7) states that the illumination over the sample has random fluctuation with variance equal to the mean intensity, characteristics typified by Poisson statistics. The superscripts on $R, E_{P}$, and e highlight that random noise sources change between acquisitions.

The combined stray and background light, $T$, is assumed to be slowly changing, and because of the source encoding [Eq. (6)], $T$ is independent of $H$ [8]. As each row of the positive and negative encodings is taken in quick succession we make the approximation $R \approx R^{+} \approx R^{-}$. Thus taking the difference between positive and negative encoding parts gives

$$
\mathbf{a}=\mathbf{a}^{+}-\mathbf{a}^{-}=\alpha_{s} R H \mathbf{p}+\mathbf{e}_{\mathbf{a}, P}+\mathbf{e},
$$

where $\mathbf{e}=\mathbf{e}^{+}-\mathbf{e}^{-}$is the total additive noise and

$$
\mathbf{e}_{\mathbf{a}, P}=\alpha_{s}\left(E_{P}^{+} H^{+}-E_{P}^{-} H^{-}\right) \mathbf{p}
$$

is the total Poisson photon noise.

\section{Decoding and Error}

Application of the inverse Hadamard transform to the acquired spectra gives

$$
\begin{aligned}
\hat{\mathbf{p}}= & \frac{1}{N} H \mathbf{a}=\frac{\alpha_{s}}{N} H R H \mathbf{p} \\
& +\frac{\alpha_{s}}{N} H\left(E_{P}^{+} H^{+}-E_{P}^{-} H^{-}\right) \mathbf{p}+\frac{1}{N} H \mathbf{e} .
\end{aligned}
$$

The last term in Eq. (10) is the reduced additive noise, as discussed in Subsection 2.A. Due to the subtraction operation, the variance of the last term is $\sigma^{2}=2 \sigma_{0}^{2}$. The first term in Eq. (10) is the reconstructed pixel values but now contaminated by a bias. If the light source drifts over time then the bias corrupts the relative magnitude of the entries in $\hat{\mathbf{p}}$. If the light source does not drift then, as stated, $R=$ $\langle r\rangle I$ and

$$
\frac{\alpha_{s}}{N} H\langle r\rangle I H=\alpha_{s}\langle r\rangle .
$$

Thus when the light source does not drift then the bias is a constant.

The second term in Eq. (10) is the photon noise. If we were to illuminate the entire sample then the photon noise MSE is $\alpha_{s}\langle r\rangle_{j} N\langle p\rangle$, where $\langle p\rangle$ is the mean pixel value and $\langle r\rangle_{j}$ is the light intensity at the time that the $j$ th acquisition is taken. Recall that each positive encoding pattern (row in $\mathrm{H}^{+}$) illuminates a subset of the pixels and the corresponding negative encoding from $\mathrm{H}^{-}$illuminates the complement subset. Thus there exists the fraction $0<$ $\delta_{j}<1$ such that the photon noise MSE of the $j$ th acquisition of the positive encoding is $\left(\sigma_{j . P}^{+}\right)^{2}=$ $\delta_{j} \alpha_{s}\langle r\rangle_{j} N\langle p\rangle$ and the negative encoding $\left(\sigma_{j, P}^{-}\right)^{2}=$ $\left(1-\delta_{j}\right) \alpha_{s}\langle r\rangle_{j} N\langle p\rangle$. The photon noise MSE $\sigma_{j, \mathbf{a}, P}^{2}$ of the $j$ th acquisition in Eq. (8) is then

$$
\sigma_{j, \mathbf{a}, P}^{2}=\left(\sigma_{j, P}^{+}\right)^{2}+\left(\sigma_{j, P}^{-}\right)^{2}=\alpha_{s}\langle r\rangle_{j} N\langle p\rangle .
$$

The Hadamard decoding averages the error values (with sign flipping due to the negative entries in $H$ ) and it reduces the random noise MSE by a factor of $N$. The MSE of the estimates due to photon noise at decoding [Eq. (10)] is therefore

$$
\sigma_{\hat{\mathbf{p}}, P}^{2}=\alpha_{s}\langle r\rangle\langle p\rangle .
$$

We contrast this with straightforward pointwise acquisition of image spectra, where each pixel $p_{i}$ is individually sampled at time $t$. Each pointwise measurement is followed by a background measurement, obtained by setting the encoding to zero. The 
background measurement is subsequently subtracted from the corresponding pointwise measurement. Thus for the pointwise acquisition if we sample $N$ pixels then in total $2 N$ samples are required, the same number as the Hadamard encoding. Obtaining a zero encoding measurement with each pointwise measurement is necessary to mitigate the effect of background and baseline drift. Assuming even illumination across the sample the photon noise MSE for individual pixel acquisition is

$$
\sigma_{\text {point }, i}^{2}=\alpha_{s}\langle r\rangle_{i} p_{i}
$$

Comparing Eqs. (13) and (14) indicates that Hadamard multiplexing has on average no effect on the MSE contribution due to photon noise. Previous authors [2,3] showed that Hadamard multiplexing results in a MSE increase of $\approx 2$ (SNR reduction of $\approx 1 / \sqrt{2}$ ) in the photon noise dominant case. However, those analyses used Hadamard S-matrix encoding [1]. Full Hadamard encoding alleviates this detrimental effect of the S-matrix approach but still does not produce an improvement over pixelwise acquisition. References $[2,3]$ did not separate illumination from pixel values, whereas the source modulation paradigm makes the separation of illumination effects from pixel variation explicit. Also the analysis of [3] made the simplification $p_{i} \approx\langle p\rangle$ (a reasonable assumption for spectral dimension multiplexing), which we do not. Hassler et al. [17] obtained substantially the same result as Eqs. (1) and (14) under the context of fluorescence imaging and starting from a model considering photon noise per pixel.

The total random noise variances for both pointwise and Hadamard imaging, respectively, $\sigma_{\text {point }, t}^{2}$ and $\sigma_{t}^{2}$, are

$$
\begin{gathered}
\sigma_{\text {point }, t, i}^{2}=\alpha_{s}\langle r\rangle_{i} p_{i}+\sigma^{2}, \\
\sigma_{t}^{2}=\alpha_{s}\langle r\rangle\langle p\rangle+\frac{1}{N} \sigma^{2} .
\end{gathered}
$$

Neglecting the bias term due to light drift, the SNRs for pointwise and Hadamard imaging are therefore

$$
\begin{gathered}
\mathrm{SNR}_{\text {point }, i}^{2}=\frac{\left(\alpha_{s}\langle r\rangle_{i} p_{i}\right)^{2}}{\alpha_{s}\langle r\rangle_{t} p_{i}+\sigma^{2}}, \\
\mathrm{SNR}_{i}^{2}=\frac{N\left(\alpha_{s}\langle r\rangle p_{i}\right)^{2}}{N \alpha_{s}\langle r\rangle\langle p\rangle+\sigma^{2}} .
\end{gathered}
$$

Figure 2 shows theoretical SNR boosts with respect to the light source intensity, $r$, with $\alpha_{s}=1$, $N=256$, and, purely for illustrative purposes, $p_{i}=$ 0.5 for all $i$. The curves displayed are of the theoretical SNR boost,

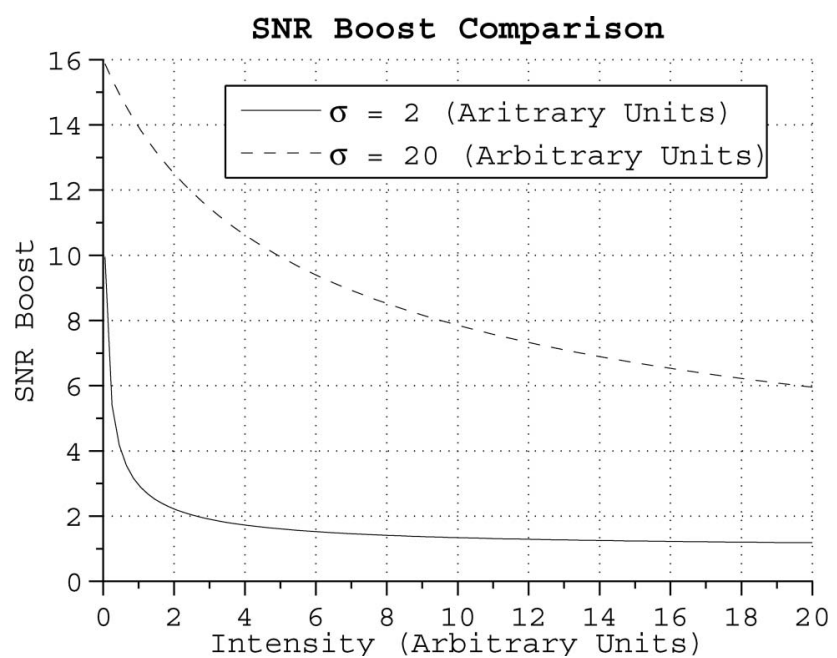

Fig. 2. Theoretical SNR boosts as a function of light source intensity for two different values of mean squared error of the additive noise.

$$
\text { boost }_{i}=\frac{\mathrm{SNR}_{i}}{\mathrm{SNR}_{\mathrm{point}, i}}=\sqrt{N \frac{\alpha_{s}\langle r\rangle_{i} p_{i}+\sigma^{2}}{N \alpha_{s}\langle r\rangle\langle p\rangle+\sigma^{2}}}
$$

for a low and a moderate value of $\sigma$. The SNR boost is greatest when $\sigma^{2}$ dominates $\left.\left(\sigma^{2}\right\rangle\langle r\rangle\right)$, thus the SNR boost is delivered when most needed. As the signal increases (when $\langle r\rangle>\sigma^{2}$ ) the photon noise becomes greater than the instrument noise, hence reducing the SNR boost. However the SNR itself reaches usable levels well before the SNR boost is reduced to unity. In the event that the nature of the dominant noise source is indeterminate or varying this preferential boost is demonstrably useful.

\section{Light Drift Correction}

The purpose of light drift correction is to remove the bias due to $R$ in the first term of Eq. (10). The simplest correction method for light source drift is to acquire reference spectra and divide the acquired spectra by the reference spectra before decoding. Assuming that the reference beam is acquired very quickly before or after the sample spectrum, light drift between sample and reference is negligible. The measured reference is represented by the diagonal matrix $R_{r}$ with entries

$$
\left\{R_{r}\right\}_{i, i}=\alpha_{r}\left\{R+E_{P, r}\right\}_{i, i}+\left\{\mathbf{e}_{r}\right\}_{i}
$$

where $\alpha_{r}$ is the reference optics effect, $E_{P, r}$ is the reference photon noise, and $\mathbf{e}_{r}$ is the instrument noise. The reference beam is designed to pass the maximum amount of light to the sensor, thus $\{\boldsymbol{R}\}_{i, i}=\operatorname{var}\left(\left\{\boldsymbol{E}_{P r}\right\}_{i, i}\right) \gg \operatorname{var}\left(\left\{\mathbf{e}_{r}\right\}_{i}\right)$, moreover $\{R\}_{i, i} \gg \operatorname{var}\left(\left\{E_{P, r}\right\}_{i, i}\right)^{1 / 2}$. The total error variance in the reference beam is then well approximated by the photon noise, $\sigma_{r, i}^{2}=\alpha_{r}\langle r\rangle$.

Application of the reference beam correction and then decoding gives 


$$
\hat{\mathbf{p}}=\frac{1}{N} H \frac{1}{\alpha_{r}} R^{-1}\left[\alpha_{s} R H p\right]+\mathbf{e}_{t}=\frac{\alpha_{s}}{\alpha_{r}} \mathbf{p}+\mathbf{e}_{t},
$$

where $\mathbf{e}_{t}$ is the total random error in the estimate, i.e., the noise due to instrument and photon effects combined with the reference beam noise. In a previous paper [16] we found the total random error variance (MSE) for our system considering only instrument noise. Expanding to include photon noise variance we observe that the total MSE after reference correction is

$$
\begin{aligned}
\sigma_{t}^{2} & =\frac{1}{N} \operatorname{var}\left(\frac{\mathbf{a}}{R}\right) \\
& =\frac{1}{N\left\langle R_{r}\right\rangle^{2}}\left(\sigma_{a}^{2}+\frac{\langle\mathbf{a}\rangle^{2}}{\left\langle R_{r}\right\rangle^{2}} \sigma_{r}^{2}-2 \frac{\langle\mathbf{a}\rangle}{\left\langle R_{r}\right\rangle} \sigma_{a, r}^{2}\right),
\end{aligned}
$$

where the second line in Eq. (22) uses the equation for the variance of the ratio of two variables with random error [18], $\langle\mathbf{a}\rangle$ is the expected value of Eq. (8), and $\sigma_{a, r}^{2}$ is the covariance between the sample spectra and the reference beam spectra. Substituting $\sigma_{a}^{2}=\alpha_{s}\langle r\rangle N\langle p\rangle+\sigma^{2}, \quad \sigma_{r}^{2}=\left\langle R_{r}\right\rangle=\alpha_{r}\langle r\rangle, \quad$ and $\langle\mathbf{a}\rangle=$ $\alpha_{s}\langle r\rangle N\langle p\rangle$ gives

$$
\begin{aligned}
\sigma_{t}^{2}= & \frac{1}{N\left(\alpha_{r}\langle r\rangle\right)^{2}}\left(\alpha_{s}\langle r\rangle N\langle p\rangle+\sigma^{2}\right. \\
& \left.+\frac{\alpha_{s}^{2}\langle r\rangle^{2} N^{2}\langle p\rangle^{2}}{\alpha_{r}^{2}\langle r\rangle^{2}} \alpha_{r}\langle r\rangle-2 \frac{\alpha_{s}\langle r\rangle N\langle p\rangle}{\alpha_{r}\langle r\rangle} \sigma_{a, r}^{2}\right) .
\end{aligned}
$$

Only the reference beam is chopped [16], thus the raw measurements include light from both the sample and the reference beam. The reference beam measurements are easily obtained by subtracting the sample measurement from the raw measurement. This subtraction results in a covariance of $\sigma_{a, r}^{2}=-\sigma^{2}-\alpha_{s}\langle r\rangle N\langle p\rangle$. Substituting and rearranging, the MSE of the reference corrected estimates is

$$
\sigma_{t}^{2}=\frac{N \alpha_{s}\langle r\rangle\langle p\rangle+\sigma^{2}}{N \alpha_{r}^{2}\langle r\rangle^{2}}\left(1+\frac{2 N \alpha_{s}\langle p\rangle}{\alpha_{r}}\right)+\frac{N\langle p\rangle^{2}}{\alpha_{r}\langle r\rangle} \frac{\alpha_{s}^{2}}{\alpha_{r}^{2}} .
$$

Typically the light path of any reference beam system is designed to pass as much light as possible, thus $\alpha_{r} \gg \alpha_{s}$. So, to a close approximation

$$
\sigma_{t}^{2} \approx \frac{\alpha_{s}\langle r\rangle N\langle p\rangle+\sigma^{2}}{N \alpha_{r}^{2}\langle r\rangle^{2}}
$$

The expected value of Eq. (21) for pixel $i$ is $\alpha_{s} p_{i} / \alpha_{r}$. Therefore the SNR is approximately

$$
\mathrm{SNR}_{i}^{2} \approx \frac{N\left(\alpha_{s}\langle r\rangle p_{i}\right)^{2}}{N \alpha_{s}\langle r\rangle\langle p\rangle+\sigma^{2}}
$$

The right-hand side of Eq. (26) is the same as Eq. (18), except in Eq. (18) we neglected a bias error term due to light source drift. Therefore, if the intensity of the reference beam is sufficient ( $\alpha_{r}$ dominates $\alpha_{s}$ ), then the decrease in SNR due to reference beam correction is negligible. If the bias due to light drift is significant, then the reference beam correction removes the corresponding systematic error. Clearly when $N \alpha_{s}\langle r\rangle\langle p\rangle \gg \sigma^{2}, \mathrm{SNR}^{2}$ is linearly dependent on $\langle r\rangle$. When $\sigma^{2} \gg N \alpha_{s}\langle r\rangle\langle p\rangle, \mathrm{SNR}^{2}$ is approximately $N$ times that of measuring each pixel individually.

\section{Experimental Verification}

Our prototype hyperspectral imaging system $[\underline{8}, \underline{16}]$ fits the theoretical considerations described in $\mathrm{Sec}-$ tion 2. Figure 3 shows a diagram of the optical configuration. The optical configuration is a broadband image projector with a DMD as the optical modulator. The DMD consists of a grid of small (typically $14 \mu \mathrm{m} \times 14 \mu \mathrm{m})$ mirrors that can be dynamically programmed to deflect to a nominally on or off state. The flexibility of the DMD and the broad spectral characteristics of the mirrors make the DMD a natural choice for encoding light in visible/near infrared (Vis/NIR) spectroscopy. The system projects light patterns onto a sample to be imaged while measuring the spectral response with a KES Analysis Inc. (New York, USA) 100 series diode array spectrometer. The spectrometer samples light in direct view mode, which is the entrance slit with a collection lens directed at the sample to collect reflected light from the entire sample object region. Photon noise occurs in the light source, and instrument noise occurs in

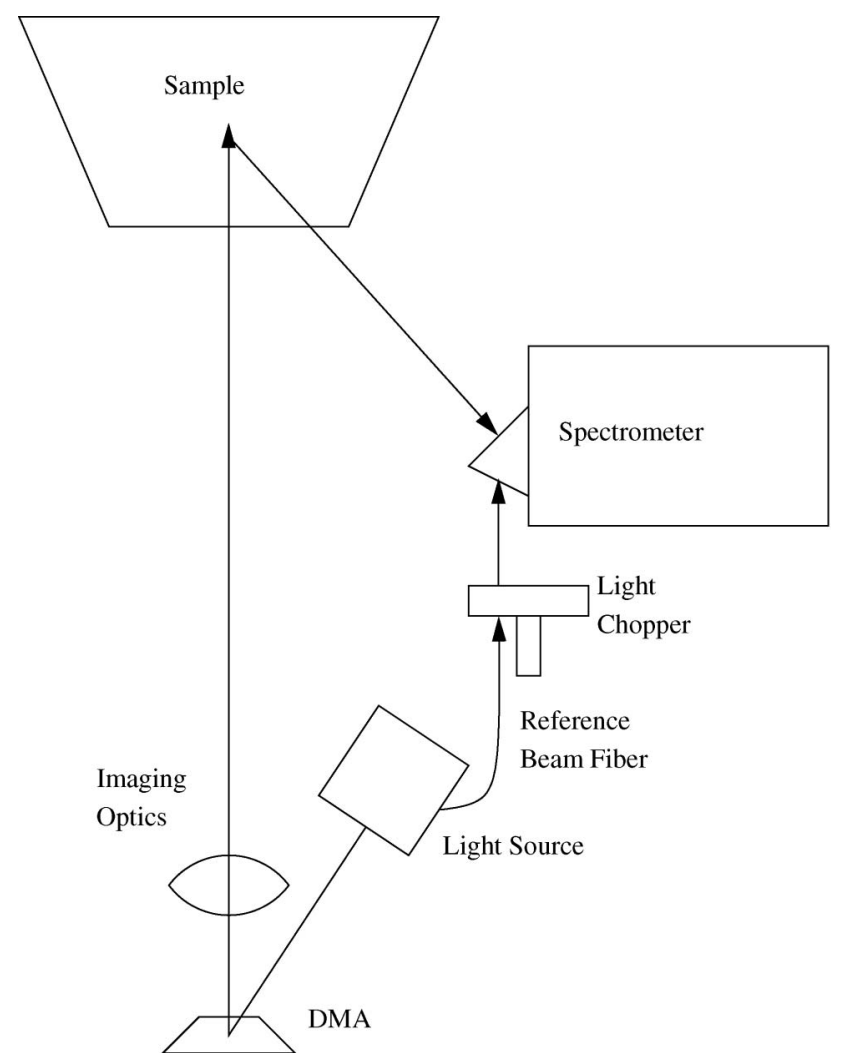

Fig. 3. Plan view diagram of the optics. Arrows indicate the path of light collected by the spectrometer. 
the optical sensor and hardware. The light source can drift, and some background/stray light is always present.

\section{A. Experimentation}

The SNR boost was quantified by the following procedure [16]:

1. Acquire a set of $16 \times 16(N=256)$ Hadamard hyperspectral images.

2. Concurrently acquire images by pointwise (per-pixel) illumination of pixels.

3. Compute the SNR image for both Hadamard and pointwise images.

4. Compute the mean SNR spectrum for each SNR image.

5. Calculate the ratio of Hadamard to pointwise SNR to give the SNR boost.

A Spectralon (Labsphere, North Sutton, N.H., USA) white tile was used for the SNR measurements. The Spectralon tile has the highest diffuse reflectivity known to us in the Vis/NIR region, thus the photon statistic effect on the SNR boost is maximized.

Hyperspectral images were taken of two simple samples. The first sample consists of polystyrene with two strips of rimu wood (Dacrydium cupressinum) inlaid diagonally. The second sample consists of acrylic with PCB fiberglass overlaid on the right-hand side. To demonstrate the usability of the spectra we segmented the images. A singular value decomposition (SVD) [19] was used to extract the principal components, and simple thresholding of the SVD scores was used to perform the segmentation.

\section{Results and Discussion}

Figures 4-6 show spectra and images from the simple example application. A different image of the diagonal wood and polystyrene object, acquired by S-matrix encoding, appeared previously [8]. This is a new image of the same object acquired by $\mathrm{H}$-matrix

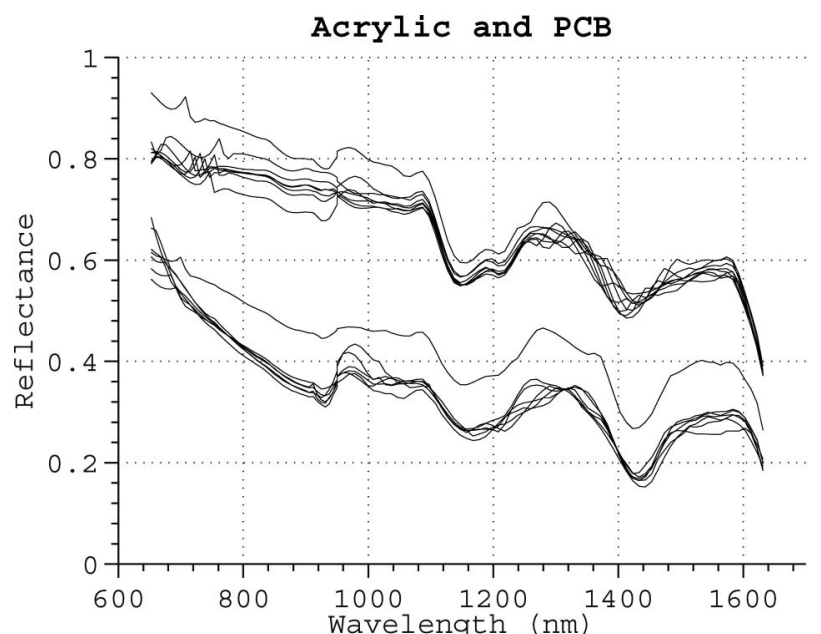

Fig. 4. Spectra from the eighth row of the acrylic and PCB fiberglass image, distinguishable by the gross intensity difference, i.e., acrylic has more reflectance.

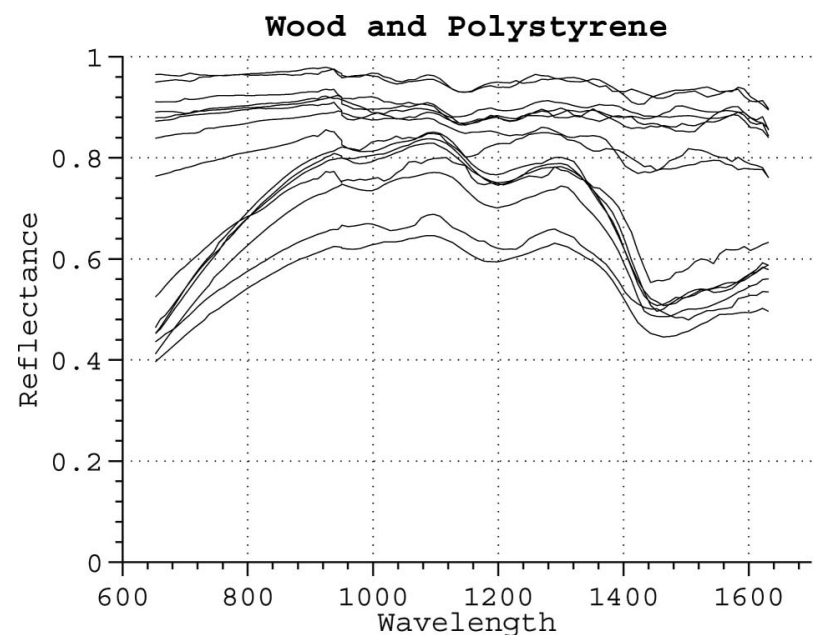

Fig. 5. Spectra from the eighth row of the wood and polystyrene image, distinguishable by their respective spectral shape. Wood has pronounced absorbance bands near $600 \mathrm{~nm}$ and around $1500 \mathrm{~nm}$.

encoding. These application images demonstrate the usability of the data generated by the imager. The spectra have been white tile corrected to remove spatial effects due to the imaging optics. Some edge effects are visible in the lower right-hand region of both images. In Figs. 4 and 5 the spectra have been cut off at $600 \mathrm{~nm}$ due to excessive noise. In each case the difference between the groups is clear. At all wavelengths the extra attenuation due to the PCB fiberglass over the acrylic is apparent. At $1533 \mathrm{~nm}$ the wood inlay appears as two dark diagonal regions in the polystyrene. Segmentation of the images by

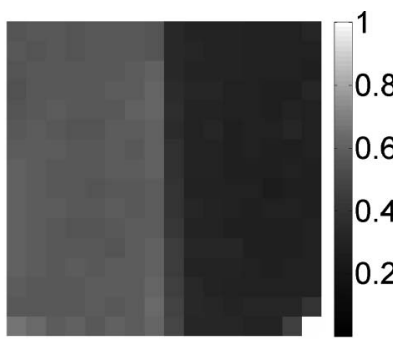

(a)

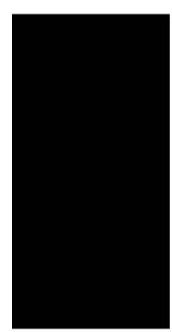

(c)

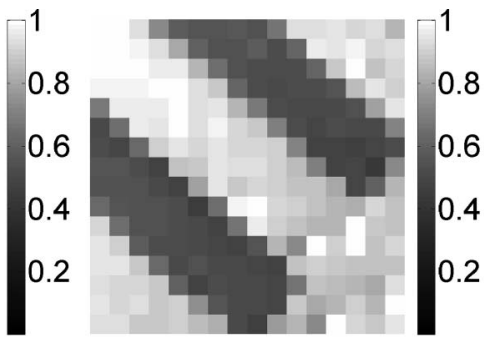

(b)

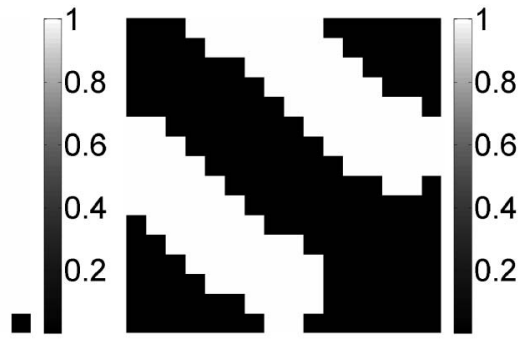

(d)
Fig. 6. Images from the simple application. (a) Image of the acrylic and PCB fiberglass sample at $1533 \mathrm{~nm}$. (b) Image of the wood and polystyrene sample at $1533 \mathrm{~nm}$. (c) Segmented image classifying the bare acrylic in (a) as black and the PCB fiberglass over acrylic as white. (d) Segmented image classifying the wood in (b) as white and polystyrene as black. 


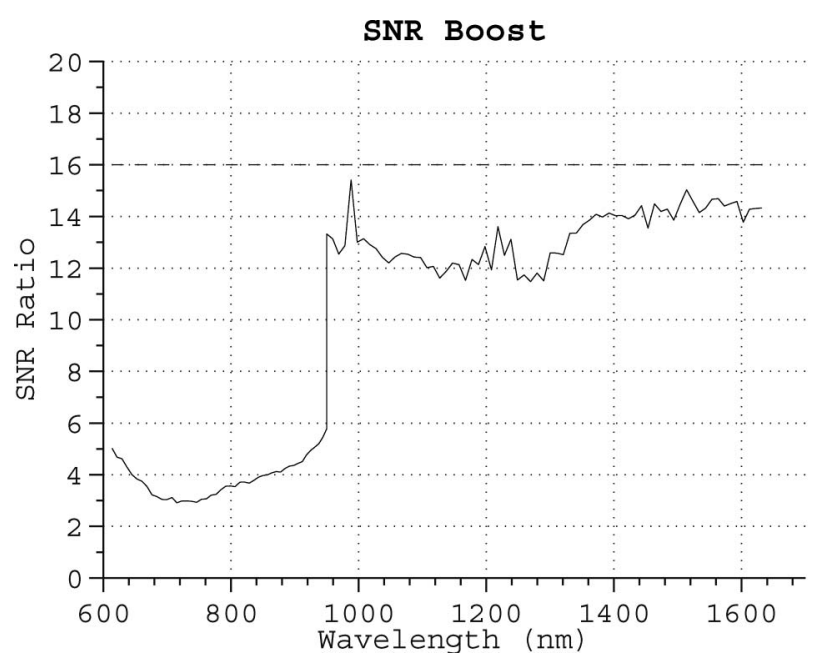

Fig. 7. SNR boost via Hadamard multiplexing. The horizontal line is the theoretical boost of 16 .

thresholding the SVD scores distinguishes between the two groups of each image, with the notable exception of the edge effect in the acrylic-PCB image.

Figure 7 shows the mean SNR boost for the imaging system. As $N=256$, the theoretical maximum boost is $\sqrt{N}=16$. The shape of the SNR boost is similar to that of previous work [16] using S-matrix complement encoding. The light level and detector sensitivity both vary with wavelength. Hence the dominant noise source varies with wavelength. The SNR boost is about the theoretical maximum at the highest wavelengths, coinciding with the region of minimal illumination from the light source. The SNR boost reaches a minimum of about 3 around $700 \mathrm{~nm}$, which coincides with the spectral peak of the light source, where the photon noise is greater that the instrument noise. The discontinuity in the SNR boost near $950 \mathrm{~nm}$ is due to the use of two types of sensor in the spectrometer for the different spectral regions. The sensor for the longer wavelength region has lower sensitivity, therefore greater sensor noise. Thus the Hadamard advantage in the longer wavelength region is better.

A factor not explicitly discussed in the theoretical framework is the photon Poisson noise contribution associated with the background and stray light. This noise is independent of the encoding and so is reduced by multiplexing. Mathematically this noise is represented in the random additive noise term. However, strictly when the background/stray light effect is significant, the source-modulated multiplexing reduces the overall influence of photon noise on the SNR.

The full Hadamard multiplexing resulted in a greater overall SNR boost than S-matrix complement encoding reported in [16] by approximately a factor of 2. S-matrix complement encoding uses complementary positive and negative encoding matrices similar to the $H^{+}$and $H^{-}$patterns used here. The theoretical SNR boost improvement in the H-matrix method over S-matrix complement encoding for random additive noise is $\sqrt{2}$. When Poisson noise is the only noise source, the S-matrix SNR boost is reduced by a further $\sqrt{2}$ to give a maximum total theoretical SNR boost improvement of 2 . Complement encoding deals implicitly with background/stray light. If instead we use S-matrix multiplexing without complement encoding then explicit measurement and subtraction of the background/stray light is necessary. Furthermore to mitigate any effect due to drift in the background/stray then it is necessary to obtain a background measurement for every encoded measurement, similar to the pointwise acquisition (Subsection 2.C). The SNR boost in complement S-matrix encoding is $\sqrt{2}$ of plain S-matrix encoding, so the H-matrix boost is in turn a factor of 2 more than plain S-matrix encoding.

\section{Conclusion}

The theory and practice of full H-matrix Hadamard imaging with additive and multiplicative noise and bias error sources was presented. The theory is general and adaptable to different multiplexing situations by reconsideration of the noise and error sources. The multiplicative noise in our case is photon noise following Poisson statistics, the multiplicative bias is due to light drift. We showed that, unlike S-matrix multiplexing, H-matrix multiplexing has, on average, no effect on the photon noise variance. Nevertheless the lack of reduction in photon noise limits the Hadamard SNR boost. This limit occurs when the light level, hence the SNR, is high. The result was interpreted as a preferential noise reduction, which is useful in cases where the dominant noise source is varying or indeterminate.

The H-matrix Hadamard imaging theory was applied to a spatially modulated hyperspectral imaging system. The dominant noise source in the imaging system varied with wavelength. Thus the SNR boost also varied with wavelength. The maximum SNR boost was delivered where the SNR in the system was inherently lowest and was most needed. Simple images were acquired using the imaging system, and the spectra were processed using SVD and segmented via a threshold. The example images demonstrate the usability of the hyperspectral images produced by the imaging system.

The authors acknowledge the statistical advice and review by Martin Upsdell and the fabrication of equipment by Keith Hill and Brian Atkins and thank the anonymous reviewers for thier insight. L. Streeter acknowledges the financial support of a New Zealand Foundation for Research, Science and Technology (FRST) Enterprise Scholarship and a University of Waikato Doctoral Scholarship.

\section{References}

1. M. Harwit and N. Sloan, Hadamard Transform Optics (Academic, 1979).

2. R. Damaschini, "Limitation of the multiplex gain in HADAMARD transform spectroscopy," Pure Appl. Opt. 2, 173-177 (1993). 
3. G. Nitzsche and R. Riesenberg, "Noise, fluctuation, and HADAMARD-transform spectrometry," Proc. SPIE 5111, 273-282 (2003).

4. A. Wuttig, "Optimal transformations for optical multiplex measurements in the presence of photon noise," Appl. Opt. 44, 2710-2719 (2005).

5. N. Ratner, Y. Schechner, and F. Goldberg, "Optimal multiplexed sensing: bounds, conditions and a graph theory link," Opt. Express 15, 17072-17092 (2007).

6. D. S. Davis, "Multiplexed imaging by means of optically generated Kronecker products: 1. the basic concept," Appl. Opt. 34, 1170-1176 (1995).

7. W. Fateley, R. Hammaker, R. DeVerse, R. Coifman, and F. Geshwind, "The other spectroscopy: demonstration of a new de-dispersion imaging spectrograph," Vib. Spectrosc. 29, 163-170 (2002).

8. L. Streeter, G. Burling-Claridge, M. Cree, and R. Künnemeyer, "Visible/near infrared hyperspectral imaging via spatial illumination source modulation," J. Near Infrared Spectrosc. 15, 395-399 (2007).

9. Q. Hanley, P. Verveer, D. Arndt-Jovin, and T. Jovin, "Threedimensional spectral imaging by HADAMARD transform spectroscopy in a programmable array microscope," J. Microsc. 197, 5-14 (2000).

10. R. DeVerse, R. Hammaker, and W. Fateley, "Realization of the HADAMARD multiplex advantage using a programmable optical mask in a dispersive flat-field near-infrared spectrometer," Appl. Spectrosc. 54, 1751-1758 (2000).
11. W. Fateley, R. Hammaker, and R. DeVerse, "Modulations used to transmit information in spectrometry and imaging," J. Mol. Struct. 550-551, 117-122 (2000).

12. R. DeVerse, R. Hammaker, and W. Fateley, "Hadamard transform raman imagery with a digital micro-mirror array," Vib. Spectrosc. 19, 177-186 (1999).

13. D. Takhar, J. Laska, M. Wakin, M. Duarte, D. Baron, S. Sarvotham, K. Kelly, and R. Baraniuk, "A new compressive imaging camera architecture using optical-domain compression," Proc SPIE 6065, 606509 (2006).

14. M. Wakin, J. Laska, M. Duarte, D. Baron, S. Sarvotham, D. Takhar, K. Kelly, and R. Baraniuk, "An architecture for compressive imaging," in 2006 IEEE International Conference on Image Processing (IEEE, 2006), pp. 1273-1276.

15. L. Nguyen, B. Aazhang, and J. F. Young, "All-optical CDMA with bipolar codes," Electron. Lett. 31, 469-470 (1995).

16. L. Streeter, G. Burling-Claridge, M. Cree, and R. Künnemeyer, "Reference beam method for source modulated HADAMARD multiplexing," Proc SPIE 6812, 68160J1 (2008).

17. K. Hassler, T. Anhut, and T. Lasser, "Time-resolved HADAMARD fluorescence imaging," Appl. Opt. 44, 75647572 (2005).

18. K. Pearson, "Mathematical contributions to the theory of evolution - on a form of spurious correlation which may arise when indices are used in the measurement of organs," Proc. R. Soc. London 60, 489-498 (1897).

19. L. Eldeń, Fundamentals of Algorithms: Matrix Methods in Data Mining and Pattern Recognition (SIAM, 2007), Chap. 6. 